\title{
Neue Musik in soziologischer Perspektive: Fragen, Methoden, Probleme ${ }^{1}$
}

\section{FRANK HENTSCHEL}

Lange Zeit scheint die Auffassung geherrscht zu haben, die sogenannte E- oder Kunstmusik, als deren Gipfel die Neue Musik angesehen wurde, sei analytisch, formal und immanent musikhistorisch zu betrachten, während für die sogenannte U- oder Popmusik zunächst auf soziologische Fragestellungen und Methoden zurïckzugreifen sei. Hinter dieser Auffassung dürfte eine Ideologie musikalischer Autonomie gestanden haben, die man nicht so sehr voraussetzen als vielmehr selbst kritisch historisch und soziologisch befragen sollte. Da jede Musik und jedes ästhetische Urteil notwendig in ein komplexes Geflecht kultureller, historischer, sozialer Zusammenhänge eingebunden ist, erschiene es einem unvoreingenommenen Betrachter wohl ohnehin unzweifelhaft, dass Musik, einschließlich ihrer Rezeption, grundsätzlich sowohl einer ästhetischen als auch einer historischsoziologischen Untersuchung unterzogen werden kann und sollte.

Das Soziotop der Neuen Musik ist indes verschachtelt, die Forschungssituation dürftig. Deshalb besteht das Ziel der folgenden Überlegungen lediglich darin, Fragen aufzuwerfen, Methoden zu diskutieren und Probleme anzusprechen.

\section{Definition und Werturteil}

Es ist klar, dass am Anfang einer Untersuchung des Soziotops Neuer Musik die Frage stehen muss, was diese überhaupt sei. Nun gibt es Neue Musik mit großem N, neue Musik mit kleinem n; und es gibt «neue elektronische Musik», die ihre Wurzeln vielfach eher in der Popmusik hat. ${ }^{2}$ Außerdem sehen die Sprachregelungen mancher Festivals Alternativen vor wie aktuelle Musik (MaerzMusik), zeitgenössische Musik (Dresdner Tage für zeitgenössische Musik) oder zeitgemäße Musik (Bludenzer Tage zeitgemäßer Musik). Während der Begriff

\footnotetext{
${ }^{1}$ Der gegenwärtige Aufsatz begreift sich als eine Art Ausblick, der aus zwei kürzlich abgeschlossenen Studien hervorgegangen ist. Sie müssen daher häufiger zitiert werden: Die Wittener Tage für neue Kammermusik. Über Geschichte und Historiografie aktueller Musik, unter Mitarbeit von Andreas Domann und Almut Ochsmann, Stuttgart 2007 (BzAfMw 62) sowie Bürgerliche Ideologie und Musik. Politik der Musikgeschichtsschreibung in Deutschland 1776-1871, Frankfurt a. M. und New York 2006. - Eine gekürzte Fassung des Aufsatzes erschien in Neue Zeitschrift fur Musik 5 (2010), S. 38-42.

${ }^{2}$ Ehrler, Hanno: „Mixen, Scratchen, Komponieren. „Neue elektronische Musik“ - ein Einblick“, in: MusikTexte 88 (2001), S. 9-15. Siehe auch den Beitrag von Dahlia Borsche im vorliegenden Heft.
} 
«aktuelle» Musik mehr Offenheit signalisieren und der Ausdruck «zeitgenössische» Musik sich vermutlich wenigstens des ideologischen Ballasts des Terminus Neue Musik entledigen möchte, legt der Begriff «zeitgemäße Musik» den tatsächlich in allen Bezeichnungen mitgedachten wertenden Charakter bloß: Gemeint ist niemals einfach Musik, die in der Gegenwart entsteht und erklingt, sondern eine Musik, die der Gegenwart in spezifischer Weise angemessen, eine Musik, die heute zu erklingen legitimiert sei.

Doch wer vermag das zu entscheiden - und nach welchen Kriterien? Rein ästhetisch scheint das unmöglich zu sein. Neue Musik - oder wie immer sie genannt wird - kann nicht die fortschrittlichste sein, weil es Fortschritt in den Künsten nicht gibt; ${ }^{3}$ neue Musik kann auch nicht die wahre Musik der Gegenwart sein, weil Wahrheit keine brauchbare ästhetische Kategorie ist. ${ }^{4}$ Deshalb müssen ganz offensichtlich Ideologien zu Hilfe genommen werden, wenn der spezifische Anspruch der Neuen Musik vertreten werden soll. So ist das Phänomen Neue Musik überhaupt nur unter Heranziehung soziologischer Untersuchungen in den Griff zu bekommen. Denn es geht immer auch um die Frage, wer wem das Label Neue Musik, zeitgemäße Musik usw. samt den damit verbundenen Wertideologien zubilligt. Ob ein Festivalleiter oder ein Musikhistoriker eine Musik der Neuen Musik zuordnet oder ihr diese Zuordnung versagt, ist nicht nur eine stilistische Entscheidung, sondern ein ästhetisches Werturteil. Es geht, soziologisch, also um die Frage, welche Werte welchen Gesellschaftsgruppen aus welchen Gründen wichtig erscheinen. Die Frage, was Neue Musik ist, lässt sich daher von der Frage, wie das Soziotop Neuer Musik beschaffen ist, aus welchem Personenkreis und welchen Interessen es sich zusammensetzt, nicht trennen.

$\mathrm{Zu}$ glauben, man könne Neue Musik - hypostasiert wie eine platonische Idee unabhängig von ihren jeweiligen Trägern, ihrem Image und Selbstverständnis definieren, man könne sich sozusagen von den ideologischen Vertretern der Szene lösen, wäre ein Irrtum. Natürlich ist es denkbar, bestimmte Sorten von Musik über bestimmte gemeinsame Merkmale $\mathrm{zu}$ definieren. Doch fiele das Ergebnis nicht mit dem Soziotop Neuer Musik zusammen. Ferner müsste man den naheliegenden Nachfolgefehler vermeiden, eine Kategorie «avantgardistischer» Musik zu konstruieren. Denn vor dem Hintergrund der Tatsache, dass sich einerseits jegliche Musik im Laufe der Zeit verändert und dass andererseits ästhetische Qualität wissenschaftlich nicht erfasst werden kann, ${ }^{5}$ ist die Frage, welche Veränderung welches Parameters als hinreichend bedeutsam erachtet wird, um eine Musik von einer

\footnotetext{
${ }^{3}$ Hentschel, Bürgerliche Ideologie, a.a.O., Kapitel 3.

${ }^{4}$ Siehe dazu grundlegend Käte Hamburger: Wahrheit und ästhetische Wahrheit, Stuttgart 1979.

${ }^{5}$ Hentschel, Bürgerliche Ideologie, a.a.O., Kapitel 1.
} 
anderen als «avantgardistisch» abzugrenzen, grundsätzlich ideologisch. ${ }^{6}$ Hingegen spricht theoretisch nichts dagegen, eine wertneutrale Kategorie zu bilden, die solche Musiken stilistisch zusammenfasst. Allerdings sollte man sie nicht mit dem Namen «Neue Musik» versehen, weil dieser sozialhistorisch $\mathrm{zu}$ sehr vorbelastet, d. h. an das Selbstverständnis bestimmter Szenen gebunden ist. Beispielsweise könnte das Duo Caspar Brötzmann und FM Einheit mit Blick auf ihre Materialbehandlung ohne weiteres in dieselbe Kategorie aufgenommen werden wie Komponisten, die traditionellerweise der Neuen Musik zugerechnet werden. Doch wird man nicht davon ausgehen dürfen, dass es nur ein einziges zutreffendes Modell der Ordnung des zeitgenössischen Musiklebens geben wird, so dass je nach Perspektive einander überlagernde Kategorien Anwendung finden werden. So könnte, wenn der Historiker Improvisation ins Zentrum rückt, das Duo Brötzmann / Einheit dem Jazz zugeschlagen werden. Geht der Historiker aber stärker von der sozialen Herkunft der Musiker und ihres Publikums aus, so wird man sie der experimentellen Popmusik zuschlagen. Je nach Perspektive haben Karlheinz Stockhausen und Pink Floyd mehr miteinander zu tun als Stockhausen und Wolfgang Rihm usw.

Neue Musik zu definieren, kann daher nur bedeuten, eine sozialhistorische Gemeinschaft $\mathrm{zu}$ bestimmen, die sich durch ein bestimmtes Image, bestimmte Verhaltensweisen, gemeinsame Prämissen usw. auszeichnet. ${ }^{7}$ Erst in einem nachgeordneten Schritt lassen sich mögliche Binnendifferenzierungen jener Szene oder Szenen vornehmen, die man - je nach angewandtem methodischen Modell - der Neuen Musik zuschlägt. Beispielsweise habe ich den Eindruck, dass es Phasen der Verengung und der Öffnung gab. Nachdem in Donaueschingen und Darmstadt zwischen 1950 und 1970 eine Phase der Verengung - besonders großer Exklusivität - stattgefunden hatte, öffnete sich diese Szene der Neuen Musik in den Siebzigerjahren (Neue Einfachheit). Bei den Wittener Tagen für neue Kammermusik meine ich eine Verengung in den Neunzigerjahren beobachten zu können, die mit dem Wechsel der Festivalleitung (von Wilfried Brennecke zu Harry Vogt im Jahr 1990) zusammenfällt, während sich zum Jahr 2000 hin wieder eine - anders geartete - Öffnung vollzog, die inzwischen wieder zurückgenommen worden zu sein scheint. Obwohl Musiker aus Szenen der neuen elektronischen Musik hin und wieder in Szenen, die sich selbst der Neuen Musik zugehörig fühlen, auftreten, ${ }^{8}$ bestehen zwischen ihnen größere Unterschiede.

\footnotetext{
${ }^{6}$ Vgl. Hentschel, Wittener Tage, a.a.O., S. 171-175.

${ }^{7}$ Dies habe ich in der erwähnten Studie im Ausgang von den Wittener Tagen für neue Kammermusik getan. Wenn im Folgenden von «der» Neuen Musik die Rede ist, so wird damit auf das durch dieses Festival definierte Material Bezug genommen. Diese methodische Beschränkung ist wissenschaftlich unvermeidlich. Man kann und sollte die Forschung ausdehnen, indem man weitere Kontexte, die von den jeweiligen Trägern der Neuen Musik zugeordnet werden, einbezieht, aber man kann sie nicht vom Selbstbild ihrer Träger lösen.

${ }^{8}$ Siehe Hentschel, Wittener Tage, a.a.O., S. 188-196.
} 
Komponisten, die in Szenen der Neuen Musik präsent, aber zugleich in Abonnementskonzerten vertreten sind, wie etwa Alfred Schnittke, stellen Sonderfälle dar, die nicht unterschlagen werden dürfen. Die Verbreitung der Klanginstallationen im Laufe der Neunzigerjahre hat das Feld nochmals kompliziert, weil damit die - soziologisch m. E. deutlich anders zusammengesetzten - Szenen der modernen Kunst auf vielschichtige Weise mit denen der Neuen Musik verknüpft worden sind. (Von daher verwundert es nicht, dass beispielsweise Carsten Nicolai, der sich als Künstler auch mit Klanginstallationen befasst hat, mit Blixa Bargeld von den Einstürzenden Neubauten kooperiert.) Nicht alle Komponisten, deren Werke auf einem Festival Neuer Musik erklingen, finden beim entsprechenden Publikum Zuspruch. Gija Kantschelis Komposition Magnum Ignotum wurde 1994 in Witten sehr deutlich abgelehnt und ausgebuht. Nicht zuletzt gibt es auch Anfeindungen unter den Komponisten der Szene selbst. So wurde Helmut Lachenmann in seinen Schriften mehrfach ausfällig gegen die Kollegen György Ligeti und Krzystof Penderecki usw. ${ }^{9}$

Wie die Szenen sich mit Blick auf die im Folgenden erörterten Merkmale unterscheiden, wäre eingehendere Studien wert, die die Konstitution der Szenen und Subszenen sowie insbesondere den soziologischen Sinn ihrer In- und Exklusionen, ihrer Werte und Überzeugungen aufhellen könnten.

\section{Bildungskapital}

Neue Musik wird von Intellektuellen für Intellektuelle gemacht. Wer Neue Musik produziert oder hört, steht ganz oben in der Bildungshierarchie und blickt auf Hörer zahlreicher anderer Musiken mit Überlegenheit herab.

Das sagt sich leicht, aber hier wären Untersuchungen durchzuführen, die die Interpretation von Dokumenten aus der Szene der Neuen Musik mit empirischen Forschungen kombinieren. ${ }^{10}$ Am Beispiel der Wittener Tage für neue Kammermusik konnten jedoch einige solcher Intuitionen objektiviert werden. So ließ sich zeigen, dass für das Image der Komponisten Neuer Musik hochkulturelle Bezugspunkte konstitutiv sind. ${ }^{11}$ Die Komponisten verhandeln in ihren Werken - ihren eigenen Aussagen zufolge - existenzielle, religiöse und

\footnotetext{
${ }^{9}$ Helmut Lachenmann: Musik als existentielle Erfahrung. Schriften 1966-1995, hg. von Josef Häusler, Wiesbaden 1996, S. 29-32, 94f., 105.

${ }^{10}$ Die Erhebungen von Rainer Dollase, Michael Rüsenberg und Hans J. Stollenwerk (Demoskopie im Konzertsaal, Mainz u. a. 1986) bestätigen die Bildungsfunktion Neuer Musik in mancher Hinsicht. Da ihre Studie jedoch nicht auf Neue Musik fokussiert war, liefert sie nur erste Anhaltspunkte.

${ }^{11}$ Hentschel, Wittener Tage, a.a.O., Kapitel 4.
} 
metaphysische Inhalte; und sie verarbeiten Dichter, Künstler und Philosophen, die höchstes Ansehen genießen und auch in den gängigen Schulbuchreihen entsprechende Positionen einnehmen, deren hoher Bildungswert also durchaus im gesellschaftlich institutionalisierten Bildungssystem verankert ist. Ob die Komponisten den Begriff der E-Musik verwenden oder nicht, so verstehen sie ihre Musik jedenfalls als überaus ernst, und zwar auch in Opposition zu bloß unterhaltender Musik. Humor spielt daher kaum je eine Rolle in der Neuen Musik. (Dem widerspricht übrigens nicht, dass manche Komponisten - John Cage, Mauricio Kagel, György Ligeti, Manos Tsangaris - gerade durch ihren Humor aufgefallen sind: Sie werden von einer Szene getragen, in der Humor eine statistisch zu vernachlässigende Größe darstellt.) Erotik gehört - ganz im Gegensatz zur Popmusik - dezidiert nicht zu den üblichen Merkmalen oder Themen Neuer Musik. Auch die Selbstdarstellung - insbesondere ihre sprachliche Vermittlung - wird als Bildungsinsignie eingesetzt. Solchem Image entspricht die tatsächliche Ausbildung der Komponisten. ${ }^{12}$ Denn nahezu sämtliche Komponisten Neuer Musik haben einen Hochschulabschluss vorzuweisen, der - neben einem Musikstudium - in den meisten Fällen geisteswissenschaftliche Fächer einschließt, und zwar in der Rangfolge Musikwissenschaft, Philosophie, Literaturwissenschaft, Theologie und Kunstgeschichte. Inwieweit sich dies bei Popmusikern anders verhält, wäre zu erforschen, aber bereits ohne derartige Forschung lässt sich vermuten, dass solche biografischen Informationen für das Bild von Popmusikern nicht konstitutiv sind, während sie mit großer Selbstverständlichkeit in Biografien von Musikern des Soziotops Neuer Musik mitgeteilt werden.

Indem sich eine Gruppierung ein Image verleiht, unterscheidet sie sich einerseits von anderen Gruppierungen; andererseits stellt sie Verbindungen zu Gruppierungen her, die verwandte Merkmale aufweisen, etwa Gruppierungen der Literatur oder der bildenden Kunst, Gruppierungen Intellektueller oder des höheren Bildungswesens usw. In diesem Sinne hat das Image der Komponisten Neuer Musik einen sozialdistinktiven Charakter. ${ }^{13}$ Solche Distinktion mag relativ neutral oder sie mag mit latenten oder offenen Aggressionen einhergehen. Diese aufzuspüren, dürfte besonders schwierig sein, weil es zum Anstand gerade auch gebildeter Menschen gehört, Aggressionen nicht zu zeigen. Sie treten daher im scheinbar geschützten Umfeld am ehesten hervor. ${ }^{14}$ Mir gegenüber nannte ein Kollege, der sich viel mit Neuer Musik befasst, Popmusik einmal «Unterschichtengeräusch».

\footnotetext{
${ }^{12}$ Ebd., S. 180-188.

${ }^{13}$ Mutatis mutandis dürfte Pierre Bourdieus Klassiker (Die feinen Unterschiede. Kritik der gesellschaftlichen Urteilskraft, übers. von Bernd Schwibs und Achim Russer, Frankfurt a. M. ${ }^{9} 1997$ ) immer noch den wichtigsten Ausgangspunkt solcher Studien darstellen.

${ }^{14}$ Die Methode Georgina Borns, sich in eine Institution Neuer Musik (IRCAM) einzuschleusen, um eine möglichst ungefilterte Innenperspektive zu erlangen, war daher vorbildlich (Rationalizing Culture: IRCAM, Boulez, and the Institutionalization of the Musical Avant-Garde, Berkeley u. a. 1995).
} 
In diese Richtung wäre, ergänzt um empirische Methoden, weiter zu forschen. Zum einen wäre das Publikum zu analysieren, denn eine Musikszene konstituiert sich nicht nur aus den Komponisten und Musikern, sondern auch aus dem Publikum, das sich allein empirisch erfassen lässt. $\mathrm{Zu}$ fragen wäre, ob unterschiedliche Festivals unterschiedliche Resultate ergeben. Zwar könnte man mit einiger Gewissheit davon ausgehen, dass eng verwandte Festivals wie die Wittener Tage für neue Kammermusik, die Donaueschinger Musiktage, das UltraSchall-Festival usw. auch verwandte Programme repräsentieren und ein verwandtes, teilweise - wie ich aus eigener Erfahrung weiß - identisches, Publikum aufweisen. Doch dürfte der Vergleich mit anderen Festivals, Szenen usw. aufschlussreichere Ergebnisse erzielen. Denn soziale Gruppen werden erst in ihrer Interaktion mit und in ihrer Opposition zu anderen sozialen Gruppen mit Bedeutung versehen. (Darauf, dass sich Neue Musik vermutlich nicht allein aus sich heraus verstehen lässt, sondern seine Signifikanz zu einem großen Teil erst aus seiner Position im Geflecht der Musikszenen insgesamt bezieht, wird zurückzukommen sein.) Besonders instruktiv dürfte eine soziologische Durchleuchtung von Festivals sein, die einerseits große Ähnlichkeiten mit Festivals wie den genannten besitzen, andererseits aber auch in charakteristischer Weise von ihnen abweichen. So erschienen Untersuchungen von MaerzMusik in Berlin, von blurred edges in Hamburg oder auch dem MoersFestival aussichtsreich.

Nicht allein schriftlich fixierte oder sprachlich artikulierte Gedanken indes tragen zur sozialen Positionierung bei. Auch das Verhalten von Musikern und Publikum, einschließlich der Kleidung, die sie tragen, können soziale Signifikanz besitzen. Selbstverständlich hat man als regelmäßiger Konzert- und Festivalbesucher gewisse Vorstellungen davon, welche Kleidung charakteristisch ist, doch da es noch gar keine systematischen Untersuchungen zu dieser Frage gibt, sollte man mit Spekulationen vorsichtig sein. So viel wird man aber vermuten können, dass die Kleidung prinzipiell von Bedeutung ist, auch wenn hier möglicherweise vor längerer Zeit eine Tendenz der Nivellierung eingesetzt haben mag. Im Abendkleid würde eine Frau auf einem Konzert Neuer Musik auffallen, nicht aber in der Staatsoper. Solche Untersuchungen müssten selbstverständlich den jeweiligen zeitgeschichtlichen Kontext mit berücksichtigen. Nicht nur dürfte in den Siebzigerjahren auf Festivals Neuer Musik noch ein anderer Dresscode geherrscht haben, sondern für jenes Jahrzehnt wird auch die politische Aussagekraft von Kleidung noch ganz anders einzuschätzen sein als für die Gegenwart.

Zum Verhalten bei einem Konzert Neuer Musik gehört - wie bei anderen Konzerten sogenannter E-Musik - die Andacht der Hörerschaft. Sie signalisiert Konzentration, ganze 
Hingabe an die Musik - eine wesentlich entkörperlichte Annäherung an diese Kunst. Sie schließt Tanz, Mitsingen, Pfeifen usw. aus. Man achtet - auch wenn es sich um Alltagskleidung handelt - auf die eigene Sauberkeit, verzichtet auf Getränke und Speisen im Konzertsaal. ${ }^{15}$ Der eine - geistig konnotierte - sinnliche Genuss schließt den anderen stärker körperlich konnotierten - sinnlichen Genuss aus. Das hat freilich auch musikalische Gründe: die niedrige Lautstärke, die Feinheit der sinnlichen Vorgänge, eventuell auch die Komplexität formaler Prozesse, wie sie in der Neuen Musik gang und gäbe sind. Doch dies besagt keineswegs, dass sie daher soziologisch ohne Belang wären. Eine solche voreilige Annahme wäre der aus dem Autonomiegedanken herausgewachsenen Ideologie künstlerischer Notwendigkeit geschuldet. Stattdessen wäre zu überlegen, ob nicht die musikalischen Charakteristika selbst sozial signifikant und deutbar sein könnten. Denn es wäre nicht abwegig, in Theodor W. Adornos «Kanon des Verbotenen» ${ }^{16}$ die klangliche Materialisierung des Bildungsanspruchs zu sehen: Die Musik ist so beschaffen, dass sie «bloßen»Spaß verhindert, dass sie intellektuellen Anspruch ausstrahlt, entkörperlichte Rezeption einfordert usw. Die viel beschworene und gleichzeitig mit unterschiedlichen Mitteln - etwa Einführungsveranstaltungen, Gesprächsrunden, Schulunterricht, Radiosendungen wie «Workshop neue Musik» (WDR) - bekämpfte Isolation der Neuen Musik ist daher möglicherweise ein Konstituens der Szene: Musik, die einem breiteren Publikum gefällt, kann ihr nicht angehören, kann daher auch keine Neue Musik sein. Mit der Gründung des «Vereins für musikalische Privataufführungen» hat Arnold Schönberg vielleicht auf ein Prinzip der Neuen Musik reagiert, das ebenso wesentlich war für diese Musikrichtung wie die Zwölftontechnik. Die seit 60 Jahren immer wieder vorgebrachte, aber offenbar nie mit nachhaltigem Erfolg umgesetzte Forderung nach Vermittlung dieser Musik an ein breiteres Publikum ist daher sehr aufschlussreich: Sie zielt darauf, die Isolation dieser Musik aufzuheben, weil dies aufgrund des seinerseits in der bildungsbürgerlichen Tradition stehenden Autonomiegedankens möglich erscheint. Doch derselbe Autonomiegedanke verhindert die Einsicht in die Tatsache, dass der soziale Sinn der Neuen Musik auf ihre Isolation angewiesen ist. Der historisch die aufklärerischen Ideen der Nationalerziehung fortschreibende Wunsch nach Vermittlung ist dabei selbst ein Insignie des bildungsbürgerlichen Habitus.

Bei sämtlichen Methoden wäre darauf zu achten, dass Imagebildung und Realität auseinanderzuhalten sind: Aus der schon fast klischeehaften Rolle, die beispielsweise

\footnotetext{
${ }^{15}$ Hier sind Differenzierungen zu beachten: Bei MaerzMusik nimmt man durchaus die Flasche Bier mit in den Konzertsaal, was in Donaueschingen oder Witten nicht der Fall ist. Und diese Differenz fällt ganz offensichtlich mit dem weiteren Begriff «aktueller» Musik zusammen.

${ }^{16}$ Z. B. Rolf Tiedemann (Hg.): Philosophie der neuen Musik, Frankfurt a. M. 1978, S. 40.
} 
Hölderlin für Komponisten Neuer Musik spielt, ${ }^{17}$ geht nicht hervor, dass die Komponisten tatsächlich hauptsächlich Hölderlin - statt Comics - lesen. Und die Ausblendung popularmusikalischer Interessen und Aktivitäten in autobiografischen Darstellungen müssen nicht besagen, dass den Komponisten diese Interessen und Aktivitäten wirklich fremd sind. Aber gerade verschwiegene Tatsachen sind für die Konstitution eines Images oder einer Szene belangvoll. (Es wäre ein Irrtum zu denken, Szenen konstituierten sich nur aus tatsächlichen Umständen; nicht minder relevant sind eingebildete und suggerierte Umstände.) Besonders aufschlussreich dürfte es im Übrigen sein, der Frage nachzugehen, in welchen weiteren Szenen sich das Publikum Neuer Musik bewegt und ob es dort sein Verhalten anpasst oder nicht.

\section{Lange und kurze Dauern ${ }^{18}$}

Eine übermäßig differenzierende Analyse der Szene Neuer Musik droht, tiefere historische Wurzeln des Phänomens auszublenden, obwohl gerade sie für die Deutung einige Relevanz besitzen mögen. Das andächtige Verhalten im Konzertsaal Neuer Musik lässt sich nicht aus sich allein heraus verstehen, sondern muss in Fortsetzung des bürgerlichen Konzertwesens verstanden werden, wie es sich in der zweiten Hälfte des 18. Jahrhunderts herausbildete. Bezüglich dieser Thematik lässt sich auf eine Fülle von Forschungsarbeiten zurückgreifen. ${ }^{19}$

1770 wurde in Jena das Konzertpublikum mittels Unterschrift dazu verpflichtet, «in anständiger Kleidung zu erscheinen, sich still und sittsam zu verhalten, sich währenden Concerts alles Geträncks und Tabakrauchens und Spielens zu enthalten, niemanden von anderen Persohnen männlichen oder weiblichen Geschlechts mitzubringen.» ${ }^{20}$ Solche aufklärerisch-erzieherischen Verhaltensnormierungen hatten auch einen sozialdistinktiven Sinn. Er spiegelt sich beispielsweise in St. Juliens soziologischer Metaphorik wider, auf die er zurückgriff, als er das Konzertpublikum charakterisierte: Es sei betrübend, «dass das dünne Häuflein wahrhaft für das Edle und Erhabene glühender Kunstfreunde in der Menge sich verliere und von dem betäubenden Geschrei der Proletarien übertönt» werde. «Seitdem die Masse sich von der Führung der Kunstintelligenzen emanzipiert» habe, krieche «die junge

\footnotetext{
${ }^{17}$ Siehe Hentschel, Wittener Tage, a.a.O., S. 139-141 und 194.

${ }^{18}$ Zum Begriff der longue durée siehe Fernand Braudel: „Geschichte und Sozialwissenschaften. Die lange Dauer", in: Schriften zur Geschichte, Bd. 1: Gesellschaften und Zeitstrukturen, übers. von Gerda Kurz und Siglinde Summerer, Stuttgart 1992, S. 49-87.

${ }^{19}$ Hier genügt der Hinweis auf die Bibliografie von Hanns-Werner Heister: Artikel „Konzertwesen“, in: MGG2, Kassel u. a. 1996, Sp. 707-710.

${ }^{20}$ Zit. nach Walter Salmen: Das Konzert. Eine Kulturgeschichte, München 1988, S. 64.
} 
Brut kaum flügge und halb blind aus dem Nest». ${ }^{21}$ Die in dieser Metaphorik ebenso wie in dem angemahnten Verhaltenskodex sich reflektierende soziale Distanzierung besitzt im Konzertwesen der Gegenwart ihre Ausläufer. Dabei haben sich zweifellos Nuancierungen zugetragen: Während im Opernhaus anscheinend vor allem jene Rituale fortgeführt werden, die - mit einem allerdings klärungsbedürftigen Begriff - der bürgerlichen Repräsentation dienen, knüpft das Publikum im Bereich der Neuen Musik vor allem an die Motive von Intelligenz und Bildung an. ${ }^{22}$

Vielleicht nicht ganz so alt wie die Entstehung des bürgerlichen Konzertwesens mit seinen Verhaltensnormen ist die Verbindung von Kunstmusik und schriftlich artikulierter Theorie. Neue Musik ist in hohem Maße selbstreflexiv, und diese Eigenschaft schlägt sich in Theoriebildung nieder. Die meisten Komponisten Neuer Musik flankieren ihre im engeren Sinne musikalische Tätigkeit mit einer literarischen, indem sie sich theoretisch über ihre Musik äußern. Dies geschieht häufig im Rahmen kürzerer Statements, insbesondere in Festivalprogrammen, wobei es gleichgültig ist, ob diese Statements aus eigenem Antrieb oder auf Anregung der Festivalleiter formuliert werden. Überdies wird die Szene der Neuen Musik von den Internationalen Ferienkursen für Neue Musik in Darmstadt und ähnlichen Veranstaltungen begleitet, die die theoretische Diskussion der Neuen Musik seit über einem halben Jahrhundert pflegen. Es fällt nicht schwer, diese Verbindung kompositorischer Praxis mit theoretischer Erörterung in engen Zusammenhang mit dem sozialen Ort der Neuen Musik zu bringen: In der Theoretisierung ihres Tuns schlägt sich der intellektuelle Anspruch dieser Musik nieder.

Musiktheorie gibt es in der westlichen (oder als solche konstruierten) Tradition spätestens seit der griechischen Antike. Doch wenn man die Ursprünge derjenigen Art von Theorie aufzuspüren sucht, die sich in der Neuen Musik fortsetzt, so scheinen die Wurzeln nicht viel früher als im Umfeld von Hector Berlioz und Robert Schumann zu liegen. Während in der Antike pädagogisch-soziologische (z. B. Platon, Aristoteles) und akustische, das Tonsystem darlegende Schriften über Musik (z. B. Pseudo-Euklid, Aristoxenos) die Norm darstellten, spielte im Mittelalter die pädagogisch-soziologische Dimension überhaupt keine Rolle mehr. Sattdessen wurden akustische Inhalte um praktische Aspekte wie die Lehre der Modi oder der Notation ergänzt. In der Frühen Neuzeit kamen dann auch Lehrbücher der Komposition hinzu. Doch ästhetisch-philosophische Auseinandersetzungen mit Musik gab es

\footnotetext{
${ }^{21}$ Zit. nach Hentschel, Bürgerliche Ideologie, a.a.O., S. 98.

22 Deshalb könnte eine empirische Untersuchung des Publikums Neuer Musik zu ganz anderen Resultaten führen, als Jörg Rössel sie am Opernpublikum beobachten konnte (,Kulturelles Kapital und Musikrezeption. Eine empirische Überprüfung von Bourdieus Theorie der Kunstwahrnehmung“, in: Soziale Welt 60 [2009], S. 239-257).
} 
kaum. Wann genau und warum es dazu kam, wäre zu rekonstruieren; und es scheint nicht unwahrscheinlich, dass die philosophische Selbstreflexion der Musik ein Produkt wiederum der bürgerlichen Aufwertung der Musik zum Bildungsgut darstellt.

Dass Komponisten mit großer Selbstverständlichkeit über ihre Musik schreiben, ist aus der Vogelperspektive gesehen also eine recht neue Erscheinung. Aus dem Blickwinkel der Neuen Musik heraus betrachtet, ließe sich freilich trotzdem von einer longue durée sprechen, die Berlioz, Schumann, Wagner, Liszt, Schönberg, Webern, Stockhausen, Cage etc. in eine bruchlose Tradition einreiht. Die sprachliche Kommentierung der eigenen kompositorischen Praxis daher einseitig als ein Phänomen der Neuen Musik zu deuten, würde zu kurz greifen, doch spricht solche Kontinuität auch für eine mögliche Kontinuität der soziologischen Bedeutung der musikästhetischen Theoriebildung. Freilich müsste eine Erforschung dieser Tradition und ihrer Funktion die Wandlungen des Charakters und des Inhaltes der Schriften in den Blick nehmen. Denn eine grundlegende Kontinuität schließt keine Umschichtungen, Akzentverschiebungen und neue funktionale Nuancierungen aus. Ebenso wäre in diesem Kontext danach zu fragen, ob die Theorie-Abstinenz mancher Komponisten (etwa Pendereckis) soziologisch deutbar ist und welche Rolle Theorie in anderen Szenen spielt, etwa der «neuen elektronischen Musik», aus deren Umfeld beispielsweise das Buch Soundcultures stammt. ${ }^{23}$

Endlich ließe sich sogar der Kanon des Verbotenen, den man als Konstituens der Neuen Musik bezeichnen kann, als Sedimentierung einer älteren Tradition begreifen: Seit der Aufklärung wurde die Musik zunächst in Analogie zur Kultur- oder Zivilisationsgeschichte als Fortschrittsgeschichte gedeutet und in einem weiteren Schritt die Fortschritts- in eine Emanzipationsgeschichte uminterpretiert. $^{24}$ Die sich seit der zweiten Hälfte des 19. Jahrhunderts beschleunigenden Änderungen und Ausweitungen des musikalischen Materials könnten sich als Konsequenz des verinnerlichten Fortschrittsdranges verstehen lassen, der den Rückgriff auf Altes zunehmend verhinderte und Komponisten immer kompromissloser nach Alternativen suchen ließ. Der Kollaps des tonalen Systems bei einigen Komponisten wäre dann ursächlich auch als ein ideologiegeschichtliches Phänomen aufzufassen. $\mathrm{Zu}$ fragen bliebe hier vor allem, warum die Fortschritts- und Emanzipationsideologie für einen kleinen Kreis von Komponisten nach wie vor derart attraktiv ist wie im langen 19. Jahrhundert, wo die Erklärung keine Schwierigkeiten bereitet, und warum dieses Denken etwa in der Zeit von

\footnotetext{
${ }^{23}$ Marcus S. Kleiner und Achim Szepanski (Hg.): Soundcultures. Über elektronische und digitale Musik, Frankfurt a. M. 2003.

${ }^{24}$ Hentschel, Bürgerliche Ideologie, a.a.O., Kapitel 4.
} 
Schönberg in Opposition zum Publikum geriet (statt beispielsweise einfach zu verschwinden). ${ }^{25}$

All diese Momente weisen jedenfalls darauf hin, dass ohne historische Perspektive die soziale Funktion und Kontextualisierung der Neuen Musik nicht hinreichend wird erklärt werden können.

\section{Innere Widersprüchlichkeiten}

Die Selbstbilder und kulturellen Praktiken der Neuen Musik sind in sich höchst widerspruchsvoll. Derartige Widersprüchlichkeiten aber sind ideologiegeschichtlich und damit in aller Regel auch soziologisch besonders aufschlussreich.

a) Neue Musik kultiviert die Abweichung. Komponisten Neuer Musik sind darum bemüht, Konventionen $\mathrm{zu}$ brechen und damit Erwartungen $\mathrm{zu}$ enttäuschen. So schreibt Helmut Lachenmann: «Von revolutionärem Geist und Willen kann Musik glaubwürdig zeugen, indem sie ihren bislang geläufigen Kommunikationsbereich nicht bloß erweitert, humoristisch umstülpt oder in irgendeiner bekannten oder unbekannten Richtung verfremdet, sondern indem sie anhand konkreter Alternativen Kommunikation selbst aufs Spiel setzt und Reflexion und kritisches Verhalten nicht bequem vorexerziert, sondern mit aller Konsequenz herausfordert.» ${ }^{26}$ Doch indem der Konventionsbruch zur Norm wird, hebt er sich selbst auf und führt sich ad absurdum. Dabei handelt es sich keineswegs um eine «bloß theoretische» Beobachtung. Im Gegenteil bildet sich dieser Widerspruch in der Szene Neuer Musik direkt ab: Die Hörer, die ein Komponist wie Lachenmann faktisch erreicht, erwarten von seiner Musik genau das, was sie ihnen bietet. Es macht sich entsprechend im Konzertsaal kein Raunen breit, keine Überraschung oder Abwehrhaltung. Es wird eine Musik genossen, die die Erwartungen des Publikums erfüllt. Lachenmanns Absichten ließen sich vielleicht eher umsetzen, würde seine Musik einem Personenkreis geboten, der mit Neuer Musik nicht vertraut ist. Doch eine solche Hörerschaft kommt mit seiner Musik nicht in Berührung und würde, falls es zu einer Berührung käme, sich der Reflexion möglicherweise schlicht verweigern. Umgekehrt führte die erwähnte Komposition Kantschelis in Witten zu Buhrufen, wie ich sie in solchem Kontext noch nicht erlebt hatte und auch seitdem nicht wieder beobachten konnte. Diese Musik widersprach tatsächlich den Erwartungen des Publikums,

\footnotetext{
${ }^{25}$ Siehe dazu vor allem Wolfgang J. Mommsen: Bürgerliche Kultur und politische Ordnung. Künstler, Schriftsteller und Intellektuelle in der deutschen Geschichte 1830-1933, Frankfurt a. M. 2000, S. 158-177.

${ }^{26}$ Lachenmann, a.a.O., S. 98.
} 
indem sie zahlreiche tonale Anspielungen aufwies und Kirchenmusik über Lautsprecher einspielte. Solches gehört nicht in den Konzertsaal Neuer Musik, obwohl dieser Komposition eigentlich genau das gelang, was andere Komponisten vergeblich anstreben: die Hörhaltung des Publikums zu irritieren.

b) Peter Niklas Wilson schrieb 1990 über Neue Musik, speziell neue Kammermusik, sie bleibe eine «Sumpfblüte: Produkt einer musikalischen Subkultur». ${ }^{27}$ Die Isolation der Neuen Musik ist eine Tatsache. Wie sie gedeutet wird, ist demgegenüber eine ganz andere Frage. Eine Facette der Deutung besteht in der Zuschreibung von hohem intellektuellen Anspruch und der Vorstellung, musikgeschichtlich dem breiteren Publikum voraus zu sein. (Dass dieses breitere Publikum dies eventuell auch selbst glaubt, ist noch kein Beleg für die Triftigkeit der Auffassung.) Wilsons Deutung weist noch eine andere Nuance auf. Mit dem gewählten Begriff geht eine implizite Wertung einher: Eine Subkultur ist nicht einfach nur eine Teilkultur, sondern eine Teilkultur, die mehr oder weniger in Opposition tritt zur herrschenden Kultur. ${ }^{28}$ Neue Musik als Subkultur zu bezeichnen, suggeriert also deren Opposition zu den Normen der herrschenden Kultur, doch fragt sich, wie sich ein solches Selbstbild mit der Tatsache vereinbaren lässt, dass Neue Musik nur aufgrund der hohen Bezuschussung öffentlicher Mittel überlebensfähig ist. Dies ist bereits ein klarer Hinweis darauf, dass Neue Musik nicht von herrschenden Normen abweicht, sondern sie ganz im Gegenteil in besonderem Maße erfüllt. Die Schriftsteller, die Komponisten Neuer Musik vertonen, oder die Philosophen und Bildenden Künstler, die sie ihren Werken zugrundelegen, gehören zum Kanon unserer Kultur. ${ }^{29}$ Neue Musik tritt nicht einmal in Opposition zu demjenigen Publikum, das sie nicht konsumiert: Auch dies wäre zu untersuchen, aber m. E. spaltet sich dieses Publikum hauptsächlich in Menschen, die die Neue Musik kennen und selbstbewusst einfach nicht beachten, ohne sie indes abzuurteilen, und solche, die aufgrund von Bildungsbeflissenheit erklären, dass sie sie nicht «verstehen», zugleich aber ihren hohen Wert anerkennen, weil er autoritativ nahegelegt wird. Einem dritten Teil ist die Existenz der Neuen Musik schlicht unbekannt. Neue Musik wäre demnach eher eine spezialistische Teilkultur, die die Werte der Bildung ganz im Sinne der akzeptierten Bildungsnormen, der Leitkultur, befürwortet, auch wenn zu ihrem Selbstverständnis die ideologische Auffassung gehört, sie sei normabweichend.

\footnotetext{
${ }^{27}$ Wittener Tage für Neue Kammermusik, Programmheft, Saarbrücken 1990, S. 9.

${ }^{28}$ Vgl. Wolfgang Lipp: Artikel „Subkultur“, in: Günter Endruweit und Gisela Trommsdorff (Hg.): Wörterbuch der Soziologie, Stuttgart 22002, S. 583, ferner Werner Fuchs-Heinritz, Artikel „Subkultur“, in: ders. u.a. (Hg.): Lexikon zur Soziologie, Opladen ${ }^{3} 1995$, S. 655.

${ }^{29}$ Hentschel, Wittener Tage, a.a.O., S. 133-144.
} 
c) $\mathrm{Ob}$ der Begriff der Kulturindustrie fällt oder nicht: Die Aversion gegen die Vermarktung gehört zum Selbstverständnis der Neuen Musik. «Man kann sagen, dass sich im Bereich der Kunst das kommerzielle Denken im heutigen West-Europa nach amerikanischem Vorbild in einer früher nie da gewesenen Weise etabliert hat - oder, wie es Karlheinz Stockhausen kürzlich drastisch ausgedrückt hat: «Die berühmte deutsche Kultur ist nichts anderes als ... der letzte Coca-Cola-Abklatsch.> Mitten in unserer äußerlichen Freiheit leben wir unter einer Diktatur des Kommerzes.» So schrieb Hans Zender. ${ }^{30}$ Neue Musik will außerhalb der Kulturindustrie stehen. Die Problematik des Begriffs, der - soll er als soziologisches Instrumentarium Wert besitzen -, überhaupt erst einmal schlüssig definiert werden müsste, mag hier ausgeblendet bleiben. Aber so viel ist festzuhalten: Sobald Musik so erfolgreich ist, dass sie in größerem Maße verlegt, gesendet und gekauft wird, gehört sie offenbar der Kulturindustrie an. In der Tat scheint der Kanon des Verbotenen vor allem darauf abzuzielen, die Hörbarkeit der Musik zu erschweren. Dabei richtet sich der Verbotekanon logischerweise nach den Konventionen der populäreren Musik. Denn die Durchkreuzung dieser Konventionen sichert die Abschottung von der gescholtenen Kulturindustrie. Es scheint kein Zufall zu sein, dass eine - nicht wertend, sondern logisch - negative Definition Neuer Musik sehr viel näher liegt als eine positive: Es handelt sich um eine Musik, die Dur-MollTonalität, regelmäßige Rhythmik, eine auf dem Konsonanz-Dissonanz-Gefälle basierende Harmonik, Wiederholungen aller Art und großräumige Melodik ausgrenzt. ${ }^{31}$ Es ist schwer, sich des Eindrucks zu erwehren - und dies wäre ebenfalls eine systematische Untersuchung wert -, dass sich Neue Musik durch die Abgrenzung von ihrem Konzept der Kulturindustrie oder einfacher: von der Popmusik - definiert. All das, was populäre Musik auszeichnet und was in breiterem Umfang musikalisch akzeptiert ist, wird von der Neuen Musik systematisch negiert. Dies aber führt zu dem latenten Widerspruch, dass Neue Musik letztlich von der populären Musik abhängig ist. Ohne Popmusik keine Neue Musik - so habe ich den Gedanken an anderer Stelle auf den Punkt gebracht. ${ }^{32}$

\section{Verschwisterungsideologie}

«Aristoteles formulierte in seiner Poetik jenen genialen Gedanken, dass der Umschlag eines Charakters in sein Gegenteil eines der wichtigsten Gestaltungsmittel der griechischen

\footnotetext{
${ }^{30}$ Hans Zender: „Was kann Musik heute sein“ (1988), in: Jörn Peter Hiekel (Hg.): Die Sinne denken. Texte zur Musik 1975-2003, Wiesbaden u. a. 2004, S. 145-156, hier S. 147.

${ }^{31}$ Hentschel, Wittener Tage, a.a.O., S. 176.

${ }^{32}$ Ebd., S. 233.
} 
Tragödie darstellt. Diesen Gedanken im geometrischen und im abstrakten Sinne habe ich auf die Musik übertragen. Nehmen wir z. B. eine statische Klangfläche, die sich in horizontalen Linien darstellen lässt, und lösen diese in bewegliche Flächen auf, deren Richtung und Länge wir variieren und schließlich bis zum Punkt reduzieren. Auf diese Weise erhalten wir eine «molekulare Struktur〉, deren Entropie man steuern kann. Lassen wir die maximale Entropie der Tonhöhen über verschiedene Stufen gleich Null werden, dass es keine Ungewissheit mehr gibt, dann entspricht das im Tonhöhenbereich dem Unisono. In gleicher Weise können wir alle Parameter behandeln und damit die Spannung zwischen der vollkommenen Ordnung über verschiedene Grade der Un-Ordnung bis zur vollkommenen Unordnung ziehen.» Mit diesen Worten erläuterte Wilfried Jentzsch sein Streichquartett $72 .{ }^{33}$

Man muss die Theorien von Repräsentanten Neuer Musik ernst nehmen - gleich, ob es sich um Komponisten, künstlerische Leiter oder Journalisten usw. handelt. Sie ernst zu nehmen, heißt, danach zu fragen, inwiefern zutrifft, also wahr oder wenigstens wahrscheinlich ist, was sie schreiben. Musikwissenschaftliche Arbeiten bemühen sich hingegen in aller Regel lediglich darum, die Positionen und Überzeugungen der untersuchten Komponisten wiederzugeben oder gar «stark zu machen». Dabei liegt es fast auf der Hand, dass jene Art von Musikschrifttum Kriterien des Klaren, Deutlichen und Wahren in der Regel nicht entspricht. Die Texte zeichnen sich vielmehr durch Hermetik, Suggestivität, ungestützte Werturteile, Imponiergehabe usw. aus. Für historische und soziologische Forschung sind derartige Leerstellen, blinde Flecken oder Inkonsistenzen besonders geeignete Anknüpfungspunkte, denn gerade hinter ihnen verbergen sich häufig kulturelle Axiome, Ideologien oder gesellschaftliche Werte. Eine kritische Analyse jener Theorien könnte daher ihre sozialgeschichtliche Bedeutung am ehesten freilegen.

An dem angeführten Beispiel hieße dies, danach zu fragen, welchen Sinn es hat, wenn im Rahmen der Erläuterung eines Streichquartettes Aristoteles, Mathematik und moderne Physik beschworen werden. In der Musikwissenschaft sind solche Fragen kaum gestellt worden. Der Grund könnte in dem liegen, was ich die Verschwisterungsideologie nennen möchte: Statt die Komponisten und Werke der Neuen Musik zu einem historischen Gegenstand neben anderen zu machen, agieren Musikwissenschaftler oft als deren Verbündete. ${ }^{34}$ Gemeint ist nicht so sehr, dass die musikwissenschaftliche Betrachtung Neuer Musik grundsätzlich zu Apologien oder Elogen führe. Die Verschwisterung kann sich auch in Kritik äußern, wenn die kompositorische Umsetzung gemeinsamer Prämissen nicht überzeugt

\footnotetext{
${ }^{33}$ Wittener Tage für neue Kammermusik, Programmheft 1978, S. 23.

${ }^{34}$ Siehe dazu auch Frank Hentschel, ,Musikgeschichte im Raster der Notation“ (i. Dr.), erscheint in: LarsChristian Koch und Dörte Schmidt (Hg.) Schriftkulturen der Musik, voraussichtl. Schliengen 2011.
} 
oder wenn in der Szene der Neuen Musik Komponisten in Erscheinung treten, die bestimmte Prämissen nicht teilen. Das Problem besteht vielmehr darin, dass Musikwissenschaftler tendenziell mit denselben ästhetischen und historiografischen Prämissen die Musik analysieren, mit denen sie komponiert wurde, anstatt sich $\mathrm{zu}$ einer sozialhistorischen Verortung und Zergliederung gerade jener Prämissen aufzuschwingen.

Es liegt der Verdacht nahe, dass Neue Musik und ihre Exegeten ein gemeinsames soziales Interesse teilen, durch das ein solches Bündnis bedingt wird. Das meine ich mit der Verschwisterungsideologie. So wie der soziale Status eines Komponisten Neuer Musik vom intellektuellen Anspruch seiner Musik abhängt - und nicht vom materiellen Erfolg -, so hängt auch der des Musikwissenschaftlers - jedenfalls seiner Auffassung nach - von diesem Wert ab. Das dürfte mit ein Grund dafür sein, dass sich in Deutschland die Integration der Popmusik in die musikalische Academia so zäh gestaltete. Man wird darin eine Fortsetzung bildungsbürgerlicher Strategien und Mechanismen sehen dürfen, doch muss man sich auch der Frage stellen, wie sich angesichts der sozialen Umwälzungen der letzten 200 Jahre solche Strukturen halten konnten.

Dieser Text basiert auf einem Referat des Symposions «Soziotop Neue Musik», 11. bis 13. Juni 2010, von Klangzeitort, Institut für Neue Musik der Universität der Künste Berlin, und der Hochschule für Musik «Hanns Eisler» Berlin, mit freundlicher Unterstützung der Ernst von Siemens Musikstiftung. 